(2) Open Access Full Text Article

\title{
Analysis of Epidural Waveform to Determine Correct Epidural Catheter Placement After CSE Labor Analgesia
}

\author{
Alessandra Coccoluto (iD) \\ Giorgio Capogna (iD ${ }^{2}$ \\ Michela Camorcia ${ }^{3}$ \\ Mark Hochman (D) ${ }^{4}$ \\ Matteo Velardo (iD) \\ 'Department of Anesthesiology, \\ Policlinico Casilino, Roma, Italy; ${ }^{2}$ EESOA, \\ European School of Obstetric \\ Anesthesia, Roma, Italy; ${ }^{3}$ Department of \\ Anesthesiology, Città di Roma Hospital, \\ Roma, Italy; ${ }^{4}$ Milestone Scientific Inc, \\ Roseland, NJ, USA
}

Background: The epidural pressure is pulsatile and synchronized with arterial pulsations. Monitoring the epidural waveform has been suggested as a technique to reliably confirm the appropriate localization of the epidural catheter.

Objective: The aim of this study was to evaluate the sensitivity and specificity of the Computer Controlled Drug Delivery System with continuous pressure and waveform sensing technology (CCDDS) (CompuFlo ${ }^{\circledR}$ CathCheck $^{\mathrm{TM}}$ ) as an instrument to assess the correct placement of the catheter in the epidural space in parturients who have received combined spinal-epidural technique (CSE) for labor analgesia.

Methods: We enrolled 40 consecutive healthy patients undergoing CSE labor analgesia with successful analgesia. All the cases in which pulsatile waveforms in synchrony with heart rate were detected were considered to be true positives; all the cases in which there was the absence of pulsatile waves were followed up. If these patients had to eventually relocate or manipulate the epidural catheter, they were considered to be true negative. If the absence of pulse waves was observed in the presence of successful analgesia during labor, the patients were considered to be false negatives.

Results: Pulsatile waveforms synchronous with heart rate were observed in 33 cases associated with adequate analgesia. In 5 cases, the pulsatile waveforms were absent due to unilateral analgesia or catheter occlusion (true negatives). In 2 cases, the patients had effective analgesia but we were not able to observe a distinct pulsatile waveform. The pressure waveform analysis through the epidural catheter had a sensitivity of $95 \%$, a positive predictive value of $100 \%$, a specificity of $100 \%$ and a negative predictive value of $60 \%$.

Conclusion: Pulsatile pressure waveform recording with CCDDS through the epidural catheter resulted in high sensitivity and positive predictive value which can help the proper placement of the epidural catheter.

Keywords: epidural analgesia, combined epidural block, epidural pulse waves

\section{Introduction}

A proper needle placement in the epidural space is essential to correctly perform the technique and also to predict the probability for the epidural injection to result in successful analgesia. The greatest verification of the accurate positioning of an epidural catheter is the occurrence of adequate analgesia (or anesthesia) and the evidence of sensory block after the injection of the local anesthetic solution.

In the combined spinal epidural (CSE) needle through needle technique, there is no practical test to confirm correct epidural catheter placement. This may become
Correspondence: Giorgio Capogna EESOA, European School of Obstetric Anesthesia, via Giulia di Gallese 15, Roma, 0015I, Italy

Email capogna.giorgio@gmail.com 
most important when, after CSE labor analgesia, an untested epidural catheter must be used to extend the anesthetic block for an unplanned or urgent cesarean section.

Unfortunately, epidural catheters may be incorrectly placed or may migrate even after initial correct placement due to the patient's movements.

Transducing the pressure occurring in the epidural space produces a characteristic waveform, which reflects heart rate and peripheral pulse waves transmitted through the dura to the epidural space. ${ }^{1,2}$ The occurrence of these pulsatile waveforms in synchrony with heart rate, obtained by transducing the epidural catheter, has been reported to be a sign confirming the epidural location of the catheter. ${ }^{3}$ The use of customary invasive blood pressure monitors and pressure transducers may, however, be problematical in labor and delivery setting, and, in all cases, they may also be considered to be an "off-label" resource, since not specifically designed for this purpose.

Most recently, Milestone Scientific has introduced the Computer Controlled Drug Delivery System ${ }^{4,5}$ with continuous pressure and waveform sensing technology (CCDDS) to detect and display the pulsatile waveform found in the epidural space, utilizing a high-resolution inline pressure sensor. ${ }^{6}$

The purpose of this prospective, single arm, open-label study was to evaluate the sensitivity and specificity of CCDDS technology in detecting epidural pulse waveforms to assess the correct placement of the catheter in the epidural space in parturients who have received CSE for labor analgesia.

\section{Methods}

The study protocol was registered at Clinical Trials.gov (Registration: NCT04240912), received the approval from the Institutional Ethics Committee (Lazio 1, Roma, Italy) and all the patients gave written informed consent. The study was conducted in accordance with the Declaration of Helsinki. The study was conducted from April to September 2020.

We enrolled in this study 40 consecutive healthy patients who requested labor analgesia. All the patients received CSE analgesia which is the routine technique for labor analgesia in our hospital. The CES technique was performed with a needle through needle technique at L3/L4 or L4/L5, using a $27 \mathrm{G}$ spinal needle and a $16 \mathrm{G}$ epidural needle (CSE Minipack, Smiths Medical, USA). After giving the spinal component of the analgesic solution $(2.5 \mathrm{mg}$ of ropivacaine with $2.5 \mu \mathrm{g}$ of sufentanil), a closed ended multiport 16 $G$ epidural catheter was threaded into the epidural space and attached to a CADD Solis Pump (Smiths Medical, USA) to deliver a programmed intermittent epidural bolus (PIEB) of $10 \mathrm{~mL}$ of the maintenance analgesic solution (ropivacaine $0.01 \%$ and sufentanil $0.5 \mu \mathrm{g} / \mathrm{mL}$ ) every hour, starting immediately after the spinal analgesic dose.

The efficacy of analgesia was evaluated by a $100 \mathrm{~mm}$ Visual Analogue Pain Scale (VAPS) $(0=$ no pain; $100=$ worst pain ever) assessed at the apex of a painful contraction. Analgesia was considered successful if the patients reported a VAPS equal or less than 10 fifteen minutes after administration of the CSE dose.

Two hours after the initial spinal analgesic dose, all the patients with successful analgesia were admitted to the study and their epidural catheter was disconnected from the PIEB pump, primed with $5 \mathrm{~mL}$ of sterile saline, and connected to the CompuFlo instrument, to record the occurrence of epidural pulsatile waveforms. The CompuFlo instrument $\left(\right.$ CompuFlo $^{\circledR} \quad$ CathCheck $^{\mathrm{TM}}$ Milestone Scientific, USA) utilizes the Computer Controlled Drug Delivery System with continuous pressure and waveform sensing (CCDDS) technology, and provides objective pressure measurements and the detection of a pulsatile pressure waveform in a single system.

The initial epidural catheter priming volume was $5 \mathrm{~mL}$ of sterile saline solution. The investigator was allowed to give incremental volumes of $5 \mathrm{~mL}$ each in the case of the absence of any EPW appearance for 10 seconds up to $20 \mathrm{~mL}$. If, however, after $20 \mathrm{~mL}$ priming volume, no EPW was detected, the case was considered as negative.

All the EPW measurements were made with the patient in the semi recumbent lateral decubitus position with the epidural catheter fixed on the patient's shoulder and directly connected to the pressure transducer of the instrument, which, in turn, was positioned on the patient's bed. The patients were advised not to speak, to breathe calmly and not to move during the measurements. Neither the instrument nor the tubing set nor the transducer was handled or touched during the recordings.

All the cases in which pulsatile waveforms in synchrony with heart rate were detected were considered to be true positives; all the cases in which there was the absence of pulsatile waves were followed up. In these patients, if the epidural catheter had to be eventually relocated or manipulated, they were considered to be true negative. Criteria for manipulation or repositioning of the epidural catheter were 
the occurrence of pain during labor analgesia due to unilateral block or absence of adequate sensory block.

If the absence of pulse waves was observed in the presence of successful analgesia during labor the patients were considered to be false negatives.

The volume of saline necessary to prime the epidural catheter $(\mathrm{mL})$ was noted.

\section{Statistical Analysis}

All the results were recorded, and data were analyzed for the specificity and sensitivity.

Sample size calculation was estimated using exact methods for a diagnostic test study.

The power analysis, based on the one-sided test, was set to detect a $99 \%$ specificity and sensitivity and required a sample of 36 observations to set $80 \%$ test power and 95\% significance level.

To evaluate the accuracy of the test we measured the Area Under the curve of the Receiver Operating Characteristic (AUC, ROC curve).

\section{Results}

Thirty-eight patients had successful analgesia after the CSE and received at least an epidural PIEB bolus before the study period and therefore were included in the data analysis. Mean (SD) age was 27.3 (6.5) years, with a mean (SD) BMI of 26.7 (1.3) and a mean (SD) 39.5 (0.8) weeks of gestation; 23 $(60 \%)$ patients were nulliparas, $15(40 \%)$ were multiparas.

Pulsatile waveforms synchronous with heart rate were observed in 33 epidural catheters associated with adequate analgesia and therefore properly located in the epidural space. In all cases, the visualization of a peak-to-peak (apexto-apex) frequency was consistent with that of the cardiovascular pulse rate periodicity, but we observed a variability in the height of the amplitude between different waveforms. The waveform classification was based on the following formula: Peak-to-Trough Vertical Height/Peak-to-Peak Horizontal Distance x $100 \%=$ Pulse Wave Ratio. When the Pulse Wave Ratio was greater than $50 \%$ it was classified as "strong", when less than $50 \%$ understood to be "weak".

We observed strong waveforms in 28 cases (84\%) and weak in 5 cases $(15 \%)$ but all had a recognizable pulsation synchronous with the heart rate.

We noticed an interference with the appearance of the pulsatile wave during deep breaths in 7 patients $(21 \%)$ but this interference did not prevent us from identifying the pulsatile wave after re-priming.

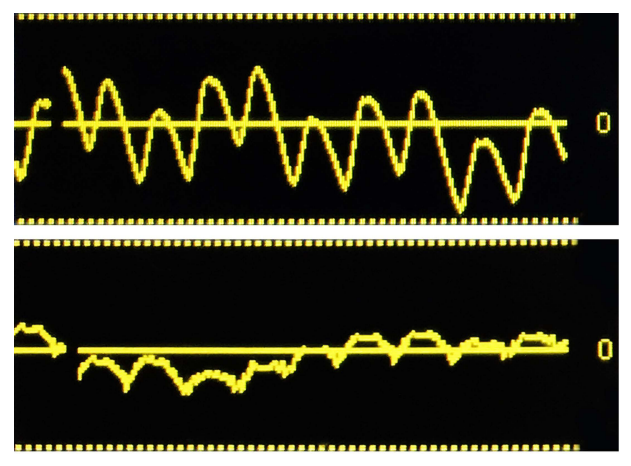

\section{A}
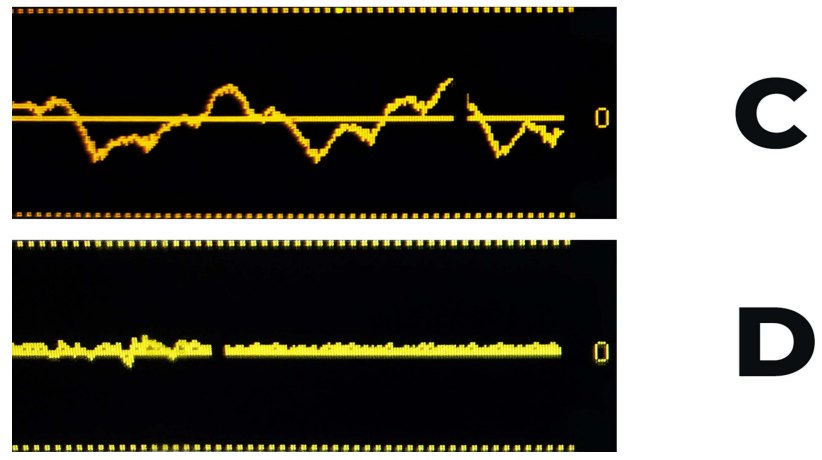

Figure I Illustration of epidural waveforms representing (A) strong, (B) weak, (C) weak with respiratory pattern and (D) absent. When accessing the presence of a positive waveform in the weak category, greater attention should be focused on details, as the vertical height of the amplitude of waveform is reduced.

In 5 cases, the pulsatile waveforms were absent. During the follow-up in two cases, these catheters were relocated because they were associated with unilateral analgesia, and in one case the occlusion of the catheter was noted and therefore these three cases were considered to be true negatives.

In 2 other cases, the patients had effective analgesia but we were not able to observe a distinct pulsatile waveform even after repeated priming, and these cases were considered as false negatives.

In Figure 1 an example of strong, weak and absent epidural waveforms is reported. An example of pulse waves during the patient's deep breathing is also depicted.

In Table 1 are reported sensitivity, specificity, positive predictive value (PPV) and negative predictive values (NVP) and accuracy values of CCDDS technology to detect epidural pulse waveforms and to assess the correct placement of the catheter in the epidural space. The pressure waveform analysis through the epidural catheter had a sensitivity of $95 \%$, a positive predictive value of $100 \%$, a specificity of $100 \%$ and a negative predictive value of $60 \%$.

In Figure 2 the Area Under the Curve (AUC) is shown, which confirms the high accuracy of our results. 
Table I Sensitivity, Specificity, Positive Predictive Value (PPV), Negative Predictive Value (NPV) and Accuracy Concerning the Ability of CCDDS Technology to Detect and Display the Pulsatile Wave Form Found in the Epidural Space

\begin{tabular}{|l|c|c|}
\hline & Value & $\mathbf{9 5 \%} \mathbf{C l}$ \\
\hline Sensitivity & 0.95 & 0.88 to 1.00 \\
Specificity & 1.00 & 1.00 to 1.00 \\
PPV & 1.00 & 1.00 to 1.00 \\
NPV & 0.60 & 0.17 to 1.00 \\
Accuracy & 0.97 & 0.93 to 1.00 \\
\hline
\end{tabular}

The epidural catheter priming volume necessary to obtain the epidural pulsatile waves ranged from 5 to $15 \mathrm{~mL}$ of saline, with a mean (SD) volume of 9.36 (4.75) $\mathrm{mL}$.

\section{Discussion}

In this study, pulsatile pressure waveform recording with the CCDDS technology through the epidural catheter resulted in high sensitivity and reliable positive predictive values.

This result was similar to that previously obtained in a completely different setting, such as with epidural catheters previously used for post-cesarean analgesia and about to be removed. ${ }^{6}$

Concerning laboring women, using a commercially available transducer for invasive pressure, Sebbag et $\mathrm{al}^{7}$ observed no epidural pressure waveforms without priming, and they attributed their findings to anatomical and physiological differences in the lumbar epidural space of parturients. On the contrary, Al-Aamri et $\mathrm{al}^{8}$ who used an invasive blood pressure transducer reported a pulsatile waveform in $96 \%$ of cases in laboring women, after

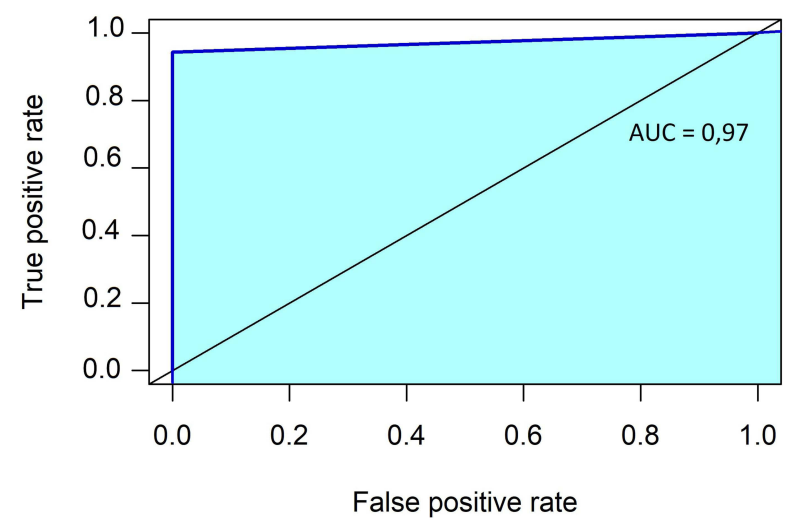

Figure 2 Area under the curve (AUC). priming the epidural space with $5 \mathrm{~mL}$ of saline, as suggested by de Medicis et al. ${ }^{9}$

However, unlike us, these previous studies in laboring women to date observed epidural waveforms through the epidural needle and not through the epidural catheter. In clinical practice, and especially in obstetrics, all the analgesic solutions are injected through the catheter and not through the needle; therefore, it is of vital importance to confirm the correct location of the catheter in the epidural space during the entire duration of labor.

During labor, an originally well-placed epidural catheter may become dislodged due to patient movement. ${ }^{10,11}$ The knowledge of the accurate position of the catheter in the epidural space whenever needed is even more important with CSE labor analgesia when an untested epidural catheter must eventually be used to extend an epidural block for an unplanned cesarean section.

Regarding technical aspects, as opposed to the pressure transducers used in the previous studies, ${ }^{3}$ the CCDDS technology, utilizing a high-resolution pressure in-line sensor system, was capable of detecting the presence of a pulsatile waveform, when present, with a high degree of sensitivity. Previous research conducted in detecting a pulsatile waveform was based upon visual inspection of a sine-wave on a medical oscilloscope display. The visual interpretation is dependent upon a clinician's subjective identification of a waveform on the screen. ${ }^{3}$ Visualization of a peak-to-peak (apex-to-apex) frequency, synchronous with that of the cardiovascular pulse rate periodicity, is consistently observed across all affirmative pulsatile pathways, while the height of the waveform amplitude is found to vary between different waveforms. It is noted that the height of the waveform amplitude is dependent on the oscilloscope setting of the vertical scale with respect to $\pm \mathrm{mm} / \mathrm{Hg}$. A unique feature of the CCDDS technology is the ability to optimize the screen by being able to change the vertical scaling of $\pm \mathrm{mm} / \mathrm{Hg}$ in real-time during the procedure thus allowing one to enhance the visualization of the waveform. Another point worth consideration, is that when accessing the presence of a positive waveform in the weak category, greater attention should be focused on details, as the vertical height of the amplitude of waveform is reduced. However, with repeated observations and experience, the weak pulsatile waveforms can also be clearly identified. Indeed, in a previous paper which used a standard pressure transducer, ${ }^{12} 50 \%$ of patients had epidural waveform of weak oscillation, whereas in our cases the majority of the oscillations were mainly strong and 
easily evident, and in only $15 \%$ the waveforms were weak. However, previous studies were performed with commercially available blood pressure transducers not specifically designed for the epidural use, and therefore less sensitive to the epidural signal.

CCDDS technology has optimized the recognition of any epidural pulsatile waveform by using a scale that can vary the sensitivity and magnitude of the pulsatile wave.

In addition, CCDDS technology utilizes standardized disposables specifically designed for the system, avoiding the use of off-label blood pressure transducers that introduce variability due to varying different setups with non-standardized components. These features are more important in obstetric anesthesia when lumbar epidurals are used, because detection of the pulse wave at the lower levels of the vertebral column has been shown to have a reduced vertical amplitude height of the pulsatile waveforms ${ }^{13}$ and therefore would ideally need a more sensitive instrument for detection.

This study has, however, some limitations. Our study was not designed to investigate whether more or less pulsatile waveforms could be revealed in the case of inadvertent placement of the epidural catheter in the subarachnoid, intravascular, or subdural place, and further studies, are required to investigate this issue.

Another theoretical limitation was the relatively low negative predictive value, but this is most likely due to the scarcity of negative cases and to the high success rate of our epidurals in our sample. In addition, the primary objective of the study was the detection of pulsatile waves when the catheter was in the epidural space and therefore the most important parameter was the positive predictive value, which confirms that the catheter is in the epidural space by the positive epidural wave.

In conclusion, pulsatile pressure waveform recording with the CCDDS technology through the epidural catheter resulted in high sensitivity and a positive predictive value. Our results can encourage the clinical use of this instrument to help in the correct identification of the catheter in the epidural space and eventually check its proper placement, which may be especially valuable in the case of CSE labor analgesia where testing the epidural catheter may be more complex.

\section{Data Sharing Statement}

The data that support the findings of this study are available from $\operatorname{EESOA}($,$) however, they are not publicly avail-$ able. Deidentified data are available from the corresponding author (Prof Giorgio Capogna, email: capogna.eesoa@gmail.com) upon reasonable request.

\section{Author Contributions}

All authors made a significant contribution to the work reported, whether that is in the conception, study design, execution, acquisition of data, analysis and interpretation, or in all these areas; they took part in drafting, revising or critically reviewing the article; gave final approval of the version to be published; have agreed on the journal to which the article has been submitted; and have agreed to be accountable for all aspects of the work.

\section{Funding}

There is no funding to report.

\section{Disclosure}

$\mathrm{GC}$ is a member of the Scientific Advisory for Milestone Inc. MH is Director, Scientific Affairs, Milestone Inc. MH also has a patent UPTO \#10646660 with royalties paid to Milestone Scientific, Inc. The authors report no other conflicts of interest in this work.

\section{References}

1. Nakamura K, Urayama K, Hoshino Y. Lumbar cerebrospinal fluid pulse wave rising from pulsations of both the spinal cord and the brain in humans. Spinal Cord. 1997;35(11):735-739. doi:10.1038/sj. sc. 3100548

2. Dawkins M. The identification of the epidural space. A critical analysis of the various methods employed. Anaesthesia. 1963;18:66-77. doi:10.1111/j.1365-2044.1963.tb13706.x

3. Hilber ND, Rijs K, Klimek M, et al. Systematic review of the diagnostic accuracy of epidural wave form analysis to identify the epidural space in surgical and labor patients. Minerva Anestesiol. 2019;85:393-400. doi:10.23736/S0375-9393.18.13089-6

4. Capogna G, Camorcia M, Coccoluto A, Micaglio M, Velardo M. Experimental validation of the CompuFlo ${ }^{\circledR}$ epidural controlled system to identify the epidural space and its clinical use in difficult obstetric cases. Int $J$ Obstet Anesth. 2018;36:28-33. doi:10.1016/j. ijoa.2018.04.008

5. Gebhard RE, Moeller-Bertram T, Dobecki D, et al. Objective epidural space identification using continuous real-time pressure sensing technology: a randomized controlled comparison with fluoroscopy and traditional loss of resistance. Anesth Analg. 2019;129(5):1319-1327. doi:10.1213/ANE.0000000000003873

6. Capogna G, Camorcia M, Berritta C, Hochman M, Velardo M. Confirmation of epidural catheter location by epidural pressure waveform recordings by the compuflo ${ }^{\circledR}$ cath-checker system. Open J Anesth. 2020;10:171-178. doi:10.4236/ojanes.2020.105015

7. Sebbag I, Qasem F, Armstrong K, Jones PM, Singh S. Waveform analysis for lumbar epidural needle placement in labour. Anaesthesia. 2016;71:984-985. doi:10.1111/anae.13540

8. Al-Aamri I, Derzi SH, Moore A, et al. Reliability of pressure waveform analysis to determine correct epidural needle placement in labouring women. Anaesthesia. 2017;72:840-844. doi:10.1111/ anae. 13872 
9. de Médicis E, Pelletier J, Martin R, Loignon MJ, Tétrault JP, Laroche L. Technical report: optimal quantity of saline for epidural pressure waveform analysis. Can J Anaesth. 2007;54:818-821. doi:10.1007/BF03021709

10. Crosby ET. Epidural catheter migration during labour: a hypothesis for inadequate analgesia. Can J Anaesth. 1990;37(7):789-793. doi:10.1007/BF03006538

11. Withington DE, Weeks SK. Repeat epidural analgesia and unilateral block. Can J Anaesth. 1994;41(7):568-571. doi:10.1007/ BF03009993
12. Hong JH, Jung SW. Analysis of epidural waveform for cervical epidural steroid injections confirmed with fluoroscopy. Medicine. 2018;97:e202. doi:10.1097/MD.0000000000010202

13. Bert RJ, Settipalle N, Tiwana E. The relationships among spinal CSF flows, spinal cord geometry, and vascular correlations: evidence of intrathecal sources and sinks. Am J Physiol Regul Integr Comp Physiol. 2019;317(3):R470-R484. doi:10.1152/ajpregu.00101.2018

\section{Publish your work in this journal}

Local and Regional Anesthesia is an international, peer-reviewed, open access journal publishing on the development, pharmacology, delivery and targeting and clinical use of local and regional anesthetics and analgesics. The journal welcomes submitted papers covering original research, basic science, clinical studies, reviews \& evaluations, guidelines, expert opinion and commentary, case reports and extended reports. The manuscript management system is completely online and includes a very quick and fair peer-review system, which is all easy to use. Visit http://www.dovepress.com/testimonials. php to read real quotes from published authors. 